\title{
Best Interpolation in a Strip II: Reduction to Unconstrained Convex Optimization*
}

\author{
ASEN L. DONTCHEV
}

Mathematical Reviews, 416 Fourth Street, Ann Arbor, MI 48107

\section{ILYA KOLMANOVSKY}

Department of Aerospace Engineering, The University of Michigan, Ann Arbor, MI 48109-2118

Received April 13, 1995; Revised October 4, 1995

\begin{abstract}
In this paper, we study the problem of finding a real-valued function $f$ on the interval $[0,1]$ with minimal $L^{2}$ norm of the second derivative that interpolates the points $\left(t_{i}, y_{i}\right)$ and satisfies $e(t) \leq f(t) \leq d(t)$ for $t \in[0,1]$. The functions $e$ and $d$ are continuous in each interval $\left(t_{i}, t_{i+1}\right)$ and at $t_{1}$ and $t_{n}$ but may be discontinuous at $t_{i}$. Based on an earlier paper by the first author [7] we characterize the solution in the case when $e$ and $d$ are linear in each interval $\left(t_{i}, t_{i+1}\right)$. We present a method for the reduction of the problem to a convex finite-dimensional unconstrained minimization problem. When $e$ and $d$ are arbitrary continuous functions we approximate the problem by a sequence of finite-dimensional minimization problems and prove that the sequence of solutions to the approximating problems converges in the norm of $W^{2,2}$ to the solution of the original problem. Numerical examples are reported.
\end{abstract}

Keywords: constrained best approximation, splines, interpolation, convex programming

\section{Introduction}

The variational characterization of interpolating cubic splines is due to Holladey [11] who showed that the unique solution of the best interpolation problem

$$
\begin{aligned}
& \left\|f^{\prime \prime}\right\|_{L^{2}[0,1]} \rightarrow \min , \\
& f \in W^{2.2}[0,1], \quad f\left(t_{i}\right)=y_{i}, \quad i=1,2, \ldots, n,
\end{aligned}
$$

is a natural cubic spline; that is, a $C^{2}$ piecewise cubic polynomial across $\left\{t_{i}\right\}$ with $f^{\prime \prime}(0)=$ $f^{\prime \prime}(1)=0$. Here $0=t_{1}<\cdots<t_{n}=1$ and $y_{i}, i=1, \ldots, n$, are given numbers, $\|\cdot\|_{L^{2}[a, b]}$ is the usual norm in $L^{2}[a, b]$, and $W^{2,2}[0,1]$ is the Sobolev space of real-valued functions with absolutely continuous first derivatives and square integrable second derivatives in $[0,1]$ equipped with the norm

$$
\|f\|_{W^{2,2}[0,1]}=\|f\|_{L^{2}[0,1]}+\left\|f^{\prime}\right\|_{L^{2}[0,1]}+\left\|f^{\prime \prime}\right\|_{L^{2}[0,1]} .
$$

*The first author was supported by National Science Foundation Grant Number DMS 9404431. The second author was supported by a François-Xavier Bagnoud doctoral fellowship and by National Science Foundation Grant Number MSS 9114630. 
The Holladey's result becomes transparent if we write the interpolation conditions as

$$
\int_{0}^{1} B_{i}(t) f^{\prime \prime}(t) d t=d_{i}, i=1,2, \ldots, n-2,
$$

where $B_{i}$ are the normalized $B$-splines of order two with support $\left[t_{i}, t_{i+2}\right]$ and $d_{i}$ are the second divided differences (see e.g., de Boor [3]). Applying the Lagrange multiplier rule (permitted since $B_{i}$ are linearly independent) one obtains that there exist Lagrange multipliers $\lambda_{i}, i=1,2, \ldots, n-2$, such that any solution of (1) is also a solution of the unconstrained optimization problem

$$
\int_{0}^{1}\left[0.5 u(t)^{2}-\sum_{i=1}^{n-2} \lambda_{i} B_{i}(t) u(t)\right] d t \rightarrow \min , \quad u \in L^{2}[0,1],
$$

where we take the square of the norm and multiply by 0.5 without changing the solution. The minimum is attained at the minimizer of the integrand, hence

$$
u(t)=f^{\prime \prime}(t)=\sum_{i=1}^{n-2} \lambda_{i} B_{i}(t)
$$

that is, the second derivative of the solution is piecewise linear across $\left\{t_{i}\right\}$ and hence the solution of (1) is a $C^{2}$ piecewise cubic polynomial.

Let us consider the problem (1) with the additional requirement $f$ be convex, i.e. $f^{\prime \prime} \geq 0$. In order to apply Lagrange multiplier rule we assume that $d_{i}>0$ (which implies the quasirelative interior condition according to Borwein and Lewis [4]). Then there exists numbers $\lambda_{1}, \ldots, \lambda_{n-2}$ such that the problem of convex best interpolation reduces to the problem

$$
\int_{0}^{1}\left[0.5 u(t)^{2}-\sum_{i=1}^{n-2} \lambda_{i} B_{i}(t) u(t)\right] d t \rightarrow \min , \quad u \geq 0, \quad u \in L^{2}[0,1] .
$$

Taking minimum of the integrand for $u \geq 0$ results in the following expression for the second derivative of the solution:

$$
u(t)=f^{\prime \prime}(t)=\max \left\{0, \sum_{i=1}^{n-2} \lambda_{i} B_{i}(t)\right\} \quad \text { for a.e. } t \in[0,1] .
$$

This result was obtained by Hornung [12] and later extended in a number of papers far beyond the interpolation setting, see Borwein and Lewis [4], Chui et al. [5], Dontchev [6], Micchelli et al. [14], Micchelli and Utreras [15]. Numerical procedures for solving convex best interpolation problem are developed in Andersson and Elfving [1], Dontchev and Kalchev [8], Irvine et al. [13].

The Lagrange duality approach has been further developed in a previous paper [7] by the first author, where the following problem was considered: find a real-valued function in 
the interval $[0,1]$ that interpolates the points $\left(t_{i}, y_{i}\right)$, has a minimal $L^{2}$ norm of the second derivative, and its graph is between the graphs of two given functions $e$ and $d$; that is,

$$
\begin{aligned}
& \left\|f^{\prime \prime}\right\|_{L^{2}[0,1]} \rightarrow \min , \\
& f\left(t_{i}\right)=y_{i}, \quad i=1,2, \ldots, n, \\
& e(t) \leq f(t) \leq d(t) \quad \text { for all } t \in[0,1],
\end{aligned}
$$

where $e$ and $d$ are continuous in each interval $\left(t_{i}, t_{i+1}\right)$ and at $t_{1}$ and $t_{n}$ but may be discontinuous at $t_{i}, i=2, \ldots,(n-1)$. In [7] necessary conditions for the solution of problem (2) were obtained which are summarized in the following lemma:

Lemma 1.1. ([7]). Assume there exists a function $\psi \in W^{2,2}[0,1]$ with $\psi\left(t_{i}\right)=$ $y_{i}, i=1,2, \ldots, n$, such that $e(t)<\psi(t)<d(t)$ for all $t \in[0,1]$. Then there exists a unique solution $f$ of problem (2). Furthermore, the second derivative $f^{\prime \prime}$ of the solution is absolutely continuous with $f^{\prime \prime}(0)=f^{\prime \prime}(1)=0$, and there exist real numbers $l_{i}, i=1, \ldots, n$, and nonnegative regular measures $\mu_{1}$ and $\mu_{2}$, supported on the sets $T_{1}=\{t \in[0,1]: f(t)=e(t)\}$ and $T_{2}=\{t \in[0,1]: f(t)=d(t)\}$, respectively, such that

$$
f^{\prime \prime \prime}(t)=l_{i}+\int_{t}^{1} d\left(\mu_{1}-\mu_{2}\right) \quad \text { for a.e. } t, t_{i} \leq t \leq 1, i=1, \ldots, n \text {. }
$$

The proof of the lemma presented in [7] uses the Hahn-Banach theorem in a way similar to deriving the maximum principle in optimal control. We note that the constants $l_{i}, i=1,2, \ldots, n$, and the measures $\mu_{j}, j=1,2$, in (3) are the Lagrange multipliers corresponding to the interpolation conditions and to the inequality constraints, respectively.

The following condition is equivalent to but more transparent than the assumption used in Lemma 1.1. Let $f(t+)$ and $f(t-)$ denote the right and, respectively, the left limit of a function $f$ at $t$. Throughout this paper we assume that the following condition holds:

$$
\begin{aligned}
& e\left(t_{1}\right)<y_{1}<d\left(t_{1}\right), \quad e\left(t_{n}\right)<y_{n}<d\left(t_{n}\right), \\
& \max \left\{e\left(t_{i}+\right), e\left(t_{i}-\right)\right\}<y_{i}<\min \left\{d\left(t_{i}+\right), d\left(t_{i}-\right)\right\}, i=2, \ldots, n-1, \\
& \inf _{t \in[0,1]}(d(t)-e(t))>0 .
\end{aligned}
$$

Based on Lemma 1.1 and on the additional assumption that the constraining functions $e$ and $d$ are linear in each interval $\left(t_{i}, t_{i+1}\right)$, it is proved in [7] that the unique solution $f$ of problem (2) is a $C^{2}$ piecewise cubic polynomial (a $C^{2}$ cubic spline) with knots $t_{i}, i=$ $1, \ldots, n$, and with no more than four additional knots in every interval $\left(t_{i}, t_{i+1}\right)$ where the solution reaches or leaves the constraints. It turns out that this result can be easily sharpened: the number of the additional knots in each interval is no more than two. Furthermore, if two knots in a given interval correspond to the same constraint, then this constraint is active between the knots. The precise result is stated in Theorem 2.1 in the following section. 
The primary purpose of this paper is to develop an approach for solving problems of the type (2) numerically. This is done in Section 3 where we present a reduction of the problem (2) to a convex unconstrained finite-dimensional optimization problem whose objective function can be computed explicitly by solving independently $n-1$ simpler optimization problems for each interval $\left[t_{i}, t_{i+1}\right]$. Thus standard algorithms can be applied that use the values of the objective function only. In Section 4 we treat the problem (2) with arbitrary continuous constraining functions $e$ and $d$. We replace $e$ and $d$ by piecewise linear and continuous functions and consider an approximating problem which can be solved by the method developed in Section 3. We then prove that the sequence of the solutions to the approximating problems converges in the norm of $W^{2,2}$ to the solution of the problem (2). In Section 5 we present numerical examples. An application of our approach to optimal motion planning in the presence of obstacles is reported in a separate paper [9].

Although quite natural in the context of curve fitting and computer-aided geometric design, the problem of best interpolation "in a strip" (2), according to the authors' knowledge, had not been considered prior to the work [7]. Opfer and Oberle [16] studied the problem of positive best interpolation, that is, the problem (1) with the additional constraint $f(t) \geq 0$. Applying a variational method based on the Du-Bois-Reimond lemma, they proved that the solution of this problem is a $C^{2}$ cubic spline with no more than two additional knots in each interval $\left[t_{i}, t_{i+1}\right]$. Fischer et al. [10] developed a local algorithm for computing a positive cubic spline in one interval $\left[t_{i}, t_{i+1}\right]$.

In a recent manuscript, L.-E. Andersson and T. Elfving [2], apparently independently of the work [7] and using a different approach obtained a characterization of the solution to problem (2) for the cases of piecewise linear and piecewise cubic constraining functions. They consider this problem as a minimum norm problem in a Hilbert space and, applying the Lagrange multiplier rule to the interpolation conditions (following [15]), reduce the problem to the projection of a certain function depending on a finite number of parameters, on the admissible set defined by the constraining functions $e$ and $d$. From this analysis they conclude that, in the case of piecewise linear constraints, the solution is a $C^{2}$ cubic spline with no more than two additional knots in each interval $\left[t_{i}, t_{i+1}\right]$. This result is stronger than the corresponding result in [7] where the solution is shown to have no more than four additional knots; however, it can be easily deduced from Lemma 1.1. For completeness we present this analysis in the proof of Theorem 2.1, where we also derive some further properties of the solution that provide the basis for our numerical approach. In addition, while both [2] and [7] consider piecewise linear and piecewise cubic constraints only, in the present paper we also consider the case of arbitrary continuous constraints.

On the computational side, the authors of [2] reduce the problem (2) to a system of nonlinear equations to which they propose to apply a Newton-type method. Here we convert (2) to an optimization problem to which one can apply a standard optimization code. These two approaches are different and it would be interesting to compare practically specific numerical implementations of them; this, however, is beyond the scope of the present paper. 


\section{A characterization of the solution}

First, we introduce some terminology. Given an interval $\left[t_{i}, t_{i+1}\right]$ and a function $f$ defined on it, we say that the point $\tau \in\left(t_{i}, t_{i+1}\right)$ is a single touching point on the constraint $e$ if $f(\tau)=e(\tau)$ and $e(t)<f(t)<d(t)$ for all $t \neq \tau, t \in\left[t_{i}, t_{i+1}\right]$. An interval $\left[\tau_{1}, \tau_{2}\right] \subset\left(t_{i}, t_{i+1}\right), \tau_{1}<\tau_{2}$, is a subarc on the constraint $e$ if $f(t)=e(t)$ for all $t \in\left[\tau_{1}, \tau_{2}\right]$ and $e(t)<f(t)<d(t)$ for all $t \in\left[t_{i}, \tau_{1}\right) \cup\left(\tau_{2}, t_{i+1}\right]$. A touching point and a subarc for the upper constraint $d$ are defined in the same way. A pair $\left(\tau_{1}, \tau_{2}\right), \tau_{1}, \tau_{2} \in\left(t_{i}, t_{i+1}\right)$, is a touching pair if $f\left(\tau_{1}\right)=e\left(\tau_{1}\right)$ and $f\left(\tau_{2}\right)=d\left(\tau_{2}\right)$, and $e(t)<f(t)<d(t)$ for all $t \neq \tau_{1}, t \neq \tau_{2}, t \in\left[t_{i}, t_{i+1}\right]$.

Theorem 2.1. Assume $e$ and $d$ are linear in each $\left(t_{i}, t_{i+1}\right), i=1, \ldots, n$. Then, there exists a unique solution $f$ of problem (2). The solution $f$ is a $C^{2}$ cubic spline (a $C^{2}$ piecewise cubic polynomial) with knots $t_{i}, i=1, \ldots, n$, and no more than $2 n-2$ additional knots in $[0,1]$ where the solution reaches or leaves the constraints. In every interval $\left[t_{i}, t_{i+1}\right]$ the following cases are possible:

1. the constraints are not active (no additional knots);

2. a single touching point on one of the constraints (one additional knot);

3. a subarc on one of the constraints (two additional knots);

4. a touching pair (two additional knots).

If in some interval $\left[t_{i}, t_{i+1}\right]$ there is a subarc on one of the constraints, then there are no subarcs on the other constraint in $\left[t_{i-1}, t_{i+2}\right]$. Moreover, $f^{\prime \prime}(0)=f^{\prime \prime}(1)=0$ and the first interval $\left[t_{1}, t_{2}\right]$ and the last interval $\left[t_{n-1}, t_{n}\right]$ do not contain subarcs.

Proof: From [7], the solution $f$ is a $C^{2}$ piecewise cubic polynomial with knots $t_{i}, i=$ $1, \ldots, n$, and with no more than four additional knots in every interval $\left(t_{i}, t_{i+1}\right)$ where the solution reaches or leaves the constraints.

We prove that if for some $\left[t_{i}, t_{i+1}\right]$ one of the constraints is active in a proper interval (with length $>0$ ), then the other constraint is nonactive in $\left[t_{i}, t_{i+1}\right]$. Let $\tau_{1}, \tau_{2}$ be two additional knots, $\tau_{1}, \tau_{2} \in\left[t_{i}, t_{i+1}\right], \tau_{1}<\tau_{2}$, let $\tau_{1}$ be the right end of a proper interval where the lower constraint $e$ is active, and let $\tau_{2}$ be the left end of an interval or a single point where the upper constraint $d$ is active, that is, $e(t)<f(t)<d(t)$ for $t \in\left(\tau_{1}, \tau_{2}\right)$. Since $f^{\prime \prime}$ is continuous in $[0,1]$, the Taylor expansion in $t \in\left[\tau_{1}, \tau_{2}\right]$ gives us

$$
f(t)=f\left(\tau_{2}\right)+f^{\prime}\left(\tau_{2}\right)\left(t-\tau_{2}\right)+\frac{1}{2} f^{\prime \prime}(\bar{\tau})\left(t-\tau_{2}\right)^{2}=d(t)+\frac{1}{2} f^{\prime \prime}(\bar{\tau})\left(t-\tau_{2}\right)^{2},
$$

for some $\bar{\tau} \in\left[t, \tau_{2}\right]$. Taking into account that $f(t)<d(t)$ for $t \in\left(\tau_{1}, \tau_{2}\right)$ we conclude that $f^{\prime \prime}(\bar{\tau})<0$ for any $\bar{\tau}$ arbitrarily close to and less than $\tau_{2}$. Hence, $f^{\prime \prime}\left(\tau_{2}\right) \leq 0$. On the other hand, $f^{\prime \prime}\left(\tau_{1}\right)=0$ since $\tau_{1}$ is the right end of an interval where $f^{\prime \prime}=0$. From Lemma 1.1, the third derivative $f^{\prime \prime \prime}$ has a jump upward at $\tau_{1}$. Hence, the linear function $f^{\prime \prime}(t)$ is strictly increasing for $\tau_{1}<t<\tau_{2}$ and $f^{\prime \prime}\left(\tau_{1}\right)=0$, thus $f^{\prime \prime}\left(\tau_{2}\right)>0$. The obtained contradiction implies that such a location of the additional knots is impossible. The proofs for the remaining cases are completely analogous. Hence, both constraints can be active in some $\left[t_{i}, t_{i+1}\right]$ in a touching pair only. 
Let $\tau_{1} \in\left[t_{i}, t_{i+1}\right]$ be the right end of a subarc on the constraint $e$ and let $\tau_{2} \in\left[t_{i+1}, t_{i+2}\right]$ be the left end of a subarc on the constraint $d$. Then $f^{\prime \prime}\left(\tau_{1}\right)=0=f^{\prime \prime}\left(\tau_{2}\right)$ and, since $\tau_{1}$ and $\tau_{2}$ are additional knots, $f^{\prime \prime}(t)>0$ for $t>\tau_{1}$ and $t$ near $\tau_{1}, f^{\prime \prime}(t)<0$ for $t<\tau_{2}$ and $t$ near $\tau_{2}$. But $f^{\prime \prime}$ is piecewise linear with one knot, $t_{i+1}$, between $\tau_{1}$ and $\tau_{2}$, a contradiction. The proof for the other case is analogous.

Lemma 1.1 implies that $f^{\prime \prime}(0)=f^{\prime \prime}(1)=0$. If $\left[\tau_{1}, \tau_{2}\right]$ is a subarc in the first interval $\left[t_{1}, t_{2}\right]$, then $f^{\prime \prime}$ is linear on $\left[t_{1}, \tau_{1}\right]$. From $f^{\prime \prime}\left(t_{1}\right)=f^{\prime \prime}\left(\tau_{1}\right)=0$ we have $f^{\prime \prime}=0$ in $\left[t_{1}, \tau_{2}\right]$. Since $\tau_{1}$ is an additional knot, $f^{\prime \prime}(t) \neq 0$ for $t<\tau_{1}, t$ near $\tau_{1}$, a contradiction. This proves the theorem.

\section{Reduction to convex optimization}

In this section, we consider problem (2), where $e$ and $d$ are linear in every interval $\left(t_{i}, t_{i+1}\right)$. First, we show that problem (2) can he rewritten as the following two-stage minimum problem:

$$
\phi(z) \rightarrow \text { Inin, } \quad z \in \mathbf{R}^{n},
$$

where

$$
\begin{aligned}
\phi(z) & =\min \left\|f^{\prime \prime}\right\|_{L^{2}}^{2} \\
f\left(t_{i}\right) & =y_{i}, \quad f^{\prime}\left(t_{i}\right)=z_{i}, \quad i=1,2, \ldots, n \\
e(t) & \leq f(t) \leq d(t) \text { for all } t \in[0,1] .
\end{aligned}
$$

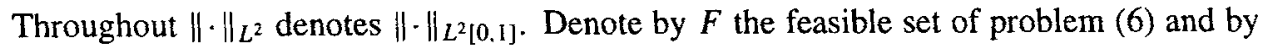
$Q(z)$ the feasible set of problem (2). Let $z_{i}^{*}=\left(f^{*}\right)^{\prime}\left(t_{i}\right), i=1,2, \ldots, n$, where $f^{*}$ is the solution of (2). From the condition (4) in Section 1 it follows that the sets $F$ and $Q(z)$, for all $z \in \mathbf{R}^{n}$, are nonempty. Since $f^{*}$ is a feasible solution for problem (2) with $z=z^{*}$, we have

$$
\left\|f^{* \prime \prime}\right\|_{L^{2}}^{2} \geq \min _{f \in Q\left(z^{*}\right)}\left\|f^{\prime \prime}\right\|_{L^{2}}^{2} \geq \min _{z \in \mathbb{R}^{n}} \min _{f \in Q(z)}\left\|f^{\prime \prime}\right\|_{L^{2}}^{2}
$$

On the other hand, a feasible solution for problem (6) is also feasible for problem (2). Hence,

$$
\left\|f^{* \prime \prime}\right\|_{L^{2}}^{2} \leq \min _{z \in \mathbf{R}^{n}} \min _{f \in Q(z)}\left\|f^{\prime \prime}\right\|_{L^{2}}^{2}
$$

Combining the above inequalities we conclude that

$$
\min _{z \in \mathbb{R}^{n}} \phi(z)=\phi\left(z^{*}\right)=\left\|f^{* \prime \prime}\right\|_{L^{2}}^{2}=\min _{f \in F}\left\|f^{\prime \prime}\right\|_{L^{2}}^{2}
$$

Thus $z^{*}$ is the unique solution of (5). 
The function $\phi$ defined in (6) can be written as

$$
\phi(z)=\sum_{i=1}^{n-1} \phi_{i}\left(z_{i}, z_{i+1}\right)
$$

where

$$
\begin{aligned}
\phi_{i}\left(z_{i}, z_{i+1}\right) & =\min \left\|f^{\prime \prime}\right\|_{L^{2}\left[t_{i}, t_{i+1}\right]}^{2} \\
f\left(t_{i}\right) & =y_{i}, \quad f^{\prime}\left(t_{i}\right)=z_{i}, \\
f\left(t_{i+1}\right) & =y_{i+1}, \quad f^{\prime}\left(t_{i+1}\right)=z_{i+1}, \quad i=1,2, \ldots, n-1, \\
e(t) & \leq f(t) \leq d(t) \text { for all } t \in\left[t_{i}, t_{i+1}\right] .
\end{aligned}
$$

It is clear that, if $f_{i}(z)$ is the solution of the $i$ th problem (7) defined on $\left[t_{i}, t_{i+1}\right]$, then the concatenation of the $f_{i}(z)$ on $[0,1]$ is the solution of (6). Thus, the function $\phi$ can be evaluated by solving $n-1$ independent problems of the form (7) on each interval $\left[t_{i}, t_{i+1}\right]$.

Theorem 3.1. The function $\phi$ is convex, coercive, and has a unique minimum in $\mathbf{R}^{n}$. Moreover, the problem (5) is Tikhonov well-posed; that is, every minimizing sequence converges to the unique solution.

Proof: The convexity of $\phi$ follows from the fact that $\phi$ is the value function of a convex minimum problem. Let $z=\lambda z^{1}+(1-\lambda) z^{2}, 0 \leq \lambda \leq 1$, let $f^{1}$ be the solution of problem (6) corresponding to $z^{1}$, and let $f^{2}$ be the solution to problem (6) corresponding to $z^{2}$. Then $\lambda f^{1}+(1-\lambda) f^{2}$ is feasible for problem (6) corresponding to $z$. We obtain

$$
\begin{aligned}
\phi\left(\lambda z^{1}+(1-\lambda) z^{2}\right) & \leq\left\|\lambda\left(f^{1}\right)^{\prime \prime}+(1-\lambda)\left(f^{2}\right)^{\prime \prime}\right\|_{L^{2}}^{2} \\
& \leq \lambda\left\|\left(f^{1}\right)^{\prime \prime}\right\|_{L^{2}}^{2}+(1-\lambda)\left\|\left(f^{2}\right)^{\prime \prime}\right\|_{L^{2}}^{2} \\
& =\lambda \phi\left(z^{1}\right)+(1-\lambda) \phi\left(z^{2}\right),
\end{aligned}
$$

hence $\phi$ is convex.

Let $f(z, \cdot)$ denote the solution to problem (6). Then, for $i=1, \ldots, n-1$,

$$
y_{i+1}-y_{i}=z_{i}\left(t_{i+1}-t_{i}\right)+\int_{t_{i}}^{t_{i+1}} \int_{t_{i}}^{\sigma_{1}} f^{\prime \prime}(z, \sigma) d \sigma d \sigma_{1} .
$$

Using the Cauchy inequality we obtain

$$
\left(t_{i+1}-t_{i}\right)\left|z_{i}\right|-\left|y_{i+1}-y_{i}\right| \leq\left|\int_{t_{i}}^{t_{i+1}} \int_{t_{i}}^{\sigma_{1}} f^{\prime \prime}(z, \sigma) d \sigma d \sigma_{1}\right| \leq\left\|f^{\prime \prime}(z)\right\|_{L^{2}} .
$$

Since the differences $y_{i+1}-y_{i}, i=1, \ldots, n-1$, do not depend on $z, \phi(z) \rightarrow \infty$ as $z \rightarrow \infty$. Thus $\phi$ is coercive. 
The minimum of problem (2), exists and is unique, hence $\phi$ attains its unique minimum in $\mathbf{R}^{n}$. Let $\left\{z_{k}\right\}$ be a minimizing sequence; that is,

$$
\phi\left(z_{k}\right) \leq \phi\left(z^{*}\right)+\delta_{k},
$$

where $z^{*}$ is the solution of problem (5) and $\delta_{k} \rightarrow 0$ as $k \rightarrow \infty$. By the coercivity of $\phi$ with respect to $z$, it follows that the sequence $\left\{z_{k}\right\}$ is bounded. Without loss of generality, assume that $z_{k} \rightarrow \bar{z}$ for some $\bar{z} \in \mathbf{R}^{n}$. Then, from the continuity of $\phi$ and the uniqueness of $z^{*}$ we obtain that $\bar{z}=z^{*}$. This proves the Tikhonov well-posedness.

In further lines we describe a procedure for computing the values of the objective function $\phi$. The value of $\phi(z), z=\left[z_{1}, z_{2}, \ldots, z_{n}\right]$, can be computed as the sum of $n-1$ independent terms $\phi_{i}\left(z_{i}, z_{i+1}\right), i=1, \ldots, n-1$, defined by (7). These computations can be accomplished in parallel.

For simplicity we demonstrate the procedure for computing the values of $\phi_{i}\left(z_{i}, z_{i+1}\right)$ on the problem with two knots $a$ and $b$ :

$$
\begin{aligned}
& \left\|f^{\prime \prime}\right\|_{L^{2}[a, b]}^{2} \rightarrow \min \\
& f(a)=c_{1}, \quad f^{\prime}(a)=s_{1}, \quad f(b)=c_{2}, \quad f^{\prime}(b)=s_{2}, \\
& e(t) \leq f(t) \leq d(t) \text { for all } t \in[a, b],
\end{aligned}
$$

where $e, d$ are linear in $\lceil a, b\rceil$ with $e(a)<c_{1}<d(a), e(b)<c_{2}<d(b)$. Clearly, the value of $\phi_{i}\left(z_{i}, z_{i+1}\right)$ is the value of the objective function of problem (8) when $c_{1}=y_{i}, c_{2}=$ $y_{i+1}, s_{1}=z_{i}, s_{2}=z_{i+1}$. From Theorem 2.1, the solution to problem (8) is a $C^{2}$ cubic spline with knots $a$ and $b$ and no more than two additional knots. The following cases are possible in $(a, b)$ : the constraints are not active, a single touching point, a subarc, a touching pair. The idea is to compute solution candidates (i.e. piecewise cubic polynomials that are feasible for problem (8), if any) corresponding to the four possible cases and then to choose the one that has the least second norm of the second derivative. We now show that the determination of solution candidates in the four possible cases reduces to elementary computations.

1. The constraints are not active: A cubic polynomial $f_{1}$ has the form

$$
f_{1}(t)=k_{1}(t-a)^{3}+k_{2}(t-a)^{2}+k_{3}(t-a)+k_{4}
$$

where the coefficients $k_{1}, k_{2}, k_{3}$, and $k_{4}$ are obtained from the boundary conditions (9) by solving a system of four linear equations.

2. A single touching point: A piecewise cubic polynomial $f_{2}$ with a single touching point $\delta \in(a, b)$ on the lower (or on the upper) constraint is written in terms of unknown parameters $k_{i}$ and $m_{i}, i=1, \ldots, 4$, as

$$
f_{2}(t)= \begin{cases}k_{1}(\delta-t)^{3}+k_{2}(\delta-t)^{2}+k_{3}(\delta-t)+k_{4}, & t \in[a, \delta], \\ m_{1}(-\delta+t)^{3}+m_{2}(-\delta+t)^{2}+m_{3}(-\delta+t)+m_{4}, & t \in[\delta, b]\end{cases}
$$


The unknowns $k_{i}, m_{i}, i=1, \ldots, 4$, and $\delta$ are determined from the boundary condition (9), the condition that $\delta$ is a single touching point, and from the continuity conditions for the function and its first and second derivatives in $[a, b]$. Once the location of the touching point $\delta$ is known, the $k_{i}$ and $m_{i}, i=1, \ldots, 4$, are obtained by solving a system of linear equations. The location of the touching point $\delta$ is determined from the condition that $f_{2}^{\prime \prime}$ is continuous in $(a, b)$ which results in a cubic equation for the value of $\delta$. This equation is solved with the help of Cardano's rule. There may be none, one or more than one solution of this equation in the interval $(a, b)$. Thus there may be up to three solution candidates with a single touching point on a given constraint.

3. A subarc: A piecewise cubic polynomial which has a subarc on the lower constraint can be described in terms of the additional knots denoted by $\delta_{1}, \delta_{2}, \delta_{1}<\delta_{2}$, and the coefficients $k_{i}, m_{i}, i=1, \ldots, 4$, as

$$
f_{3}(t)= \begin{cases}k_{1}\left(\delta_{1}-t\right)^{3}+k_{2}\left(\delta_{1}-t\right)^{2}+k_{3}\left(\delta_{1}-t\right)+k_{4}, & t \in\left[a, \delta_{1}\right], \\ e(t), & t \in\left[\delta_{1}, \delta_{2}\right], \\ m_{1}\left(-\delta_{2}+t\right)^{3}+m_{2}\left(-\delta_{2}+t\right)^{2}+m_{3}\left(-\delta_{2}+t\right)+m_{4}, & t \in\left[\delta_{2}, b\right]\end{cases}
$$

The unknowns $\delta_{1}, \delta_{2}, k_{i}, m_{i}, i=1, \ldots, 4$, are determined as the unique solution (if it exists) of a system of linear equations obtained from the boundary condition (9), the condition that $\delta_{1}$ and $\delta_{2}$ are the ends of a subarc, and the continuity of the function and its first and second derivatives in $[a, b]$. A similar procedure applies for a subarc on the upper constraint.

4. A touching pair: A piecewise cubic polynomial which has a touching pair $\left(\delta_{1}, \delta_{2}\right), \delta_{1}<$ $\delta_{2}$, is written as

$$
f_{4}(t)= \begin{cases}k_{1}\left(\delta_{1}-t\right)^{3}+k_{2}\left(\delta_{1}-t\right)^{2}+k_{3}\left(\delta_{1}-t\right)+k_{4}, & t \in\left[a, \delta_{1}\right], \\ m_{1}\left(-\delta_{1}+t\right)^{3}+m_{2}\left(-\delta_{1}+t\right)^{2}+m_{3}\left(-\delta_{1}+t\right)+m_{4}, & t \in\left[\delta_{1}, \delta_{2}\right], \\ g_{1}\left(-\delta_{2}+t\right)^{3}+g_{2}\left(-\delta_{2}+t\right)^{2}+g_{3}\left(-\delta_{2}+t\right)+g_{4}, & t \in\left[\delta_{2}, b\right]\end{cases}
$$

The unknowns $k_{i}, m_{i}, g_{i}, i=1, \ldots, 4$, and the additional knots $\delta_{1}, \delta_{2}$ are determined from the boundary condition (9), the definition of the touching pair, and the continuity of the function and its first and second derivatives in $[a, b]$. The location of the additional knots is determined from two coupled polynomial equations using any root finding method. Once the additional knots are known, the coefficients $k_{i}, m_{i}, g_{i}, i=1, \ldots, 4$, are determined from a system of four linear equations.

In each of the cases $(1-4)$ the functions $f_{i}(t)$ (if exist) are $C^{2}$ piecewise cubic polynomials in $[a, b]$ that are calculated analytically. Then we detcrminc, by finding the maxima of $f_{i}-d$ and $-f_{i}+e$, whether $f_{i}$ satisfies the inequality constraints, i.e. whether $f_{i}$ is a solution candidate.

Simple observations help to reduce the number of solution candidates that are examined when solving problem (8). For example, if there is a solution candidate for which the constraints are not active, then it is the solution and there is no need to consider other cases. If there is a solution candidate that has a subarc on a given constraint, then there is no solution candidate that has a single touching point on the same constraint or a subarc or touching point on the other constraint. Conversely, if there exists a solution candidate that 
has a single touching point on a given constraint, then there is no solution candidate that has a subarc on the same constraint or a subarc or touching point on the other constraint. If there is a solution candidate which has a touching point or a subarc, then the solution of problem (8) must have either a touching point or a subarc and there is no need to consider the case of a touching pair. All these considerations are taken into account in the procedure which solves the examples presented in Section 5.

Remark 3.1. The function $\phi$ is convex in $\mathbf{R}^{n}$, hence it is Lipschitz continuous and almost everywhere differentiable. We do not know whether this function is smooth everywhere. The domain $\mathbf{R}^{n}$ of the function $\phi$ splits into regions corresponding to all possible configurations of the additional knots; in the interior of each region the constraints do not change their status (active or nonactive). In the interior of each of the regions $\phi$ is analytic. However, we conjecture that since the marginal function of an optimization problem is typically nonsmooth, the first derivative of $\phi$ may have jumps on the boundaries of the regions.

Remark 3.2. The case when $e$ and $d$ are cubic in each interval $\left(t_{i}, t_{i+1}\right)$ can be treated analogously; here some additional analysis is needed when there are two subarcs in the same interval. An example with piecewise cubic constraints is presented in Section 5.

\section{Arbitrary continuous constraints}

In this section we consider problem (2) on the assumption that the constraints $e$ and $d$ satisfy the condition in Lemma 1.1; that is, there exists $\psi \in W^{2.2}[0,1]$ which interpolates $\left(t_{i}, y_{i}\right)$ and $e(t)<\psi(t)<d(t)$ for all $t \in[0,1]$. For simplicity we assume that $e$ and $d$ are continuous functions; the case when $e$ and $d$ are discontinuous at $t_{i}$ needs some more technical details. Denote by $f^{0}$ the unique solution of problem (2). We study the convergence of the following method for solving problem (2). First, we introduce a grid $\left\{\tau_{j}\right\}_{j=1}^{m}$ which covers $\left\{t_{i}\right\}$ and approximate $e$ and $d$ by continuous functions $e_{m}$ and $d_{m}$ that are linear in each interval $\left[\tau_{j}, \tau_{j+1}\right], j=1,2, \ldots, m-1$. Then new interpolation conditions $f\left(\tau_{j}\right)=s_{j}, \tau_{j} \neq t_{i}$, are introduced where $s_{j}$ are treated as parameters. In order to preserve the interiority condition we allow $s_{j}$ to vary between $e_{m}\left(\tau_{j}\right)+\epsilon$ and $d_{m}\left(\tau_{j}\right)-\epsilon$ for some sufficiently small $\epsilon>0$. Then we apply the procedure described in the preceding section minimizing also with respect to $s_{j}$.

The rigorous description of the method is as follows. Let $\left\{e_{m}\right\}$ and $\left\{d_{m}\right\}$ be two sequences of continuous functions that are linear in each interval $\left(\tau_{j}, \tau_{j+1}\right), j=1,2, \ldots, m, 0=$ $\tau_{1}<\tau_{2}<\cdots<\tau_{m}=1$, such that $e_{m} \rightarrow e$ and $d_{m} \rightarrow d$ uniformly in [0,1]. Without loss of generality, let $\left\{t_{1}, \ldots, t_{n}\right\} \subset\left\{\tau_{1}, \tau_{2}, \ldots, \tau_{m}\right\}$. Let $S_{m} \subset\{1,2, \ldots, m\}$ be the maximal set of indices such that if $j \in S_{m}$, then there exists $i=v_{m}(j), 1 \leq i \leq n$ such that $\tau_{j}=t_{i}$. I.et $\bar{S}_{m}=\{1,2, \ldots, m\} \backslash S_{m}$. Let $\epsilon_{0}>0$ be such that for all $m, d_{m}(t)-e_{m}(t)>\epsilon_{0}, t \in[0,1]$, and $y_{i}-e_{m}\left(t_{i}\right)>\epsilon_{0}, d\left(t_{i}\right)-y_{i}>\epsilon_{0}, i=1, \ldots, n$. Let $\epsilon_{m}<\epsilon_{0} / 4$. We consider the following problem as an approximation to problem (2):

$$
\Phi(s, z) \rightarrow \min
$$


subject to

$$
s_{j}=y_{\nu_{m}(j)}, j \in S_{m}, \quad e_{m}\left(\tau_{j}\right)+\epsilon_{m} \leq s_{j} \leq d_{m}\left(\tau_{j}\right)-\epsilon_{m}, j \in \bar{S}_{m}, \quad z \in \mathbf{R}^{m}
$$

where

$$
\begin{aligned}
& \Phi(s, z)=\min \left\|f^{\prime \prime}\right\|_{L^{2}[0,1]}^{2} \\
& f \in W^{2,2}[0,1], \\
& f\left(\tau_{j}\right)=s_{j}, \quad f^{\prime}\left(\tau_{j}\right)=z_{j}, \quad j=1,2, \ldots, m, \\
& e_{m}(t) \leq f(t) \leq d_{m}(t) \text { for all } t \in[0,1] .
\end{aligned}
$$

Problem (10) is a finite-dimensional minimization problem of the type treated in Section 3. Using an argument similar to that in Section 3 it can be verified that function $\Phi$ is convex and coercive in $z$ uniformly in $s$, for $s$ in its domain. Problem (10) has a unique solution and every minimizing sequence converges to the solution. The unique solution $f$ of problem (11) corresponding to the unique solution $(s, z)$ of problem $(10)$ is a $C^{2}$ piecewise cubic polynomial with knots $\tau_{j}, j=1, \ldots, m$, and no more than two additional knots for every interval $\left(\tau_{j}, \tau_{j+1}\right), j=1, \ldots, m-1$, where the following cases are possible: the constraints are not active, a touching point, a subarc, a touching pair. The value of $\Phi(s, z)$ can be computed as the sum of $m-1$ independent terms:

$$
\Phi(s, z)=\sum_{j=1}^{m-1} \Phi_{j}\left(s_{j}, s_{j+1}, z_{j}, z_{j+1}\right)
$$

where

$$
\begin{aligned}
\Phi_{j}\left(s_{j}, s_{j+1}, z_{j}, z_{j+1}\right) & =\min \left\|f^{\prime \prime}\right\|_{L^{2}\left[\tau_{j}, \tau_{j+1}\right]}^{2} \\
f\left(\tau_{j}\right) & =s_{j}, \quad f^{\prime}\left(\tau_{j}\right)=z_{j}, \\
f\left(\tau_{j+1}\right)=s_{j+1}, \quad f^{\prime}\left(\tau_{j+1}\right) & =z_{j+1}, \quad j=1,2, \ldots, m \\
e_{m}(t) & \leq f(t) \leq d_{m}(t) \text { for all } t \in\left[\tau_{j}, \tau_{j+1}\right] .
\end{aligned}
$$

The procedure described in Section 3 can be used for computing $\Phi_{j}$. Thus, for a given $m$ one can solve the problem (9) by applying standard optimization software. Iterating on $m$, one can then use the approximation obtained as an initial guess for the next iteration until a stopping test terminates the computations. The following theorem shows that this procedure is convergent.

Theorem 4.1. Let $e_{m}$ and $d_{m}$ be defined as above and let $\epsilon_{m} \rightarrow 0$ be a sequence such that

$$
0<\epsilon_{m}<\frac{1}{8} \max \left\{\epsilon_{0}, \min _{j=1,2, \ldots, m}\left\{\left(\left(\tau_{j+1}-\tau_{j}\right) / 8\right)^{6}\right\}\right\} \text {. }
$$


Let $f^{m}$ be the unique solution of problem (11) corresponding to the unique solution $\left(s^{m}, z^{m}\right)$ of problem (10). Then

$$
\lim _{m \rightarrow \infty}\left\|f^{m}-f^{0}\right\|_{W^{2,2}[0,1]}=0 .
$$

Proof: We first show that for every sufficiently large $m$ there exists a function $\bar{f}^{m}$ which is feasible (i.e., satisfies the interpolation conditions and the inequality constraints) for both problems (2) and (10) and, moreover,

$$
\lim _{m \rightarrow \infty}\left\|\left(\bar{f}^{m}\right)^{\prime \prime}-\left(f^{0}\right)^{\prime \prime}\right\|_{L^{2}}=0
$$

Let $g$ be feasible for problem (2) and such that $e(t)<g(t)<d(t)$ for all $t \in[0,1]$. Define $\tilde{f}^{m}=f^{0}+\delta_{m}\left(g-f^{0}\right), 0<\delta_{m}<1$. The function $\tilde{f}^{m}$ is feasible for problem (2) and $e(t)<\tilde{f}^{m}(t)<d(t)$ for all $t \in[0,1]$. We will choose $\delta_{m}$ so that $\delta_{m} \rightarrow 0$ as $m \rightarrow \infty$ and

$$
e_{m}(t) \leq \tilde{f}^{m}(t) \leq d_{m}(t)
$$

for every sufficiently large $m$ and for all $t \in[0,1]$. Let $\alpha$ be the minimal distance from $g$ to the constraints; that is

$$
0<\alpha=\min \left\{\min _{0 \leq t \leq 1}|g(t)-e(t)|, \min _{0 \leq t \leq 1}|g(t)-d(t)|\right\}
$$

and lct

$$
T=\left\{t \in[0,1]:\left|g(t)-f^{0}(t)\right| \leq \alpha / 2\right\}
$$

We select $\delta_{m}$ to be a sequence of positive numbers convergent to zero and such that

$$
\delta_{m} \geq \frac{2}{\alpha} \max \left\{\sup _{t \in[0.1]}\left|e_{m}(t)-e(t)\right|, \sup _{t \in[0,1]}\left|d_{m}(t)-d(t)\right|\right\}
$$

Let $t \in T$. We have

$$
\begin{aligned}
\tilde{f}^{m}(t)-e_{m}(t) & =f^{0}(t)+\delta_{m}\left(g(t)-f^{0}(t)\right)-e_{m}(t) \\
& =f^{0}(t)-g(t)+\delta_{m}\left(g(t)-f^{0}(t)\right)+g(t)-e(t)+e(t)-e_{m}(t) \\
& \geq-\alpha / 2+\delta_{m}\left(g(t)-f^{0}(t)\right)+\alpha+e(t)-e_{m}(t) \geq 0
\end{aligned}
$$

for $m$ sufficiently large. Analogously,

$$
\begin{aligned}
\tilde{f}^{m}(t)-d_{m}(t) & =f^{0}(t)-g(t)+\delta_{m}\left(g(t)-f^{0}(t)\right)+g(t)-d(t)+d(t)-d_{m}(t) \\
& \leq \alpha / 2+\delta_{m}\left(g(t)-f^{0}(t)\right)-\alpha+d(t)-d_{m}(t) \leq 0
\end{aligned}
$$


for $m$ sufficiently largc. Let $t \notin T$. Consider first the case $f^{0}(t)-g(t)>\alpha / 2$. We have

$$
\begin{aligned}
\tilde{f}_{m}(t)-e_{m}(t) & =f^{0}(t)-g(t)+\delta_{m}\left(g(t)-f^{0}(t)\right)+g(t)-e(t)+e(t)-e_{m}(t) \\
& \geq \alpha / 2+\delta_{m}\left(g(t)-f^{0}(t)\right)+\alpha+e(t)-e_{m}(t) \geq 0
\end{aligned}
$$

for $m$ sufficiently large. Furthermore,

$$
\begin{aligned}
\tilde{f}_{m}(t)-d_{m}(t) & =f^{0}(t)-d(t)+\delta_{m}\left(g(t)-f^{0}(t)\right)+d(t)-d_{m}(t) \\
& \leq \delta_{m}\left(g(t)-f^{0}(t)\right)+d(t)-d_{m}(t) \leq 0
\end{aligned}
$$

because of the choice of $\delta_{m}$ in (17). Finally, let $f^{0}(t)-g(t)<-\alpha / 2$. Then,

$$
\begin{aligned}
\tilde{f}^{m}(t)-e_{m}(t) & =f^{0}(t)-e(t)+\delta_{m}\left(g(t)-f^{0}(t)\right)+e(t)-e_{m}(t) \\
& \geq \delta_{m}\left(g(t)-f^{0}(t)\right)+e(t)-e_{m}(t) \geq 0
\end{aligned}
$$

because of the choice of $\delta_{m}$ in (17), and

$$
\begin{aligned}
\tilde{f}^{m}(t)-d_{m}(t) & =f^{0}(t)-g(t)+\delta_{m}\left(g(t)-f^{0}(t)\right)+g(t)-d(t)+d(t)-d_{m}(t) \\
& \leq-\alpha / 2+\delta_{m}\left(g(t)-f^{0}(t)\right)-\alpha+d(t)-d_{m}(t) \leq 0
\end{aligned}
$$

for $m$ sufficiently large. Thus (16) is proved. Clearly, $\left(\tilde{f}^{m}\right)^{\prime \prime} \rightarrow\left(f^{0}\right)^{\prime \prime} L^{2}$-strongly as $m \rightarrow \infty$.

We now construct the desired sequence $\left\{\bar{f}^{m}\right\}$. Suppose that $d_{m}\left(\tau_{j}\right)-\tilde{f}^{m}\left(\tau_{j}\right)<\epsilon_{m}$ for some $\tau_{j}, j=1, \ldots, m$, for a fixed sufficiently large $m$. Since $\epsilon_{m}<\frac{1}{8} \epsilon_{0}$, we have $j \in \bar{S}_{m}$. Let

$$
p_{j}^{m}(t)= \begin{cases}-\left(t-\tau_{j}-\gamma_{j}^{m}\right)^{3}\left(t-\tau_{j}+\gamma_{j}^{m}\right)^{3}, & t \in\left[\tau_{j}-\gamma_{j}^{m}, \tau_{j}+\gamma_{j}^{m}\right] \\ 0 & \text { otherwise }\end{cases}
$$

where $\left(\gamma_{j}^{m}\right)^{6}=\tilde{f}^{m}\left(\tau_{j}\right)-d_{m}\left(\tau_{j}\right)+2 \epsilon_{m}$. If $-e_{m}\left(\tau_{j}\right)+\tilde{f}^{m}\left(\tau_{j}\right)<\epsilon_{m}$ for some $\tau_{j}$ we take $p_{j}^{m}$ with negative sign and with $\gamma_{j}^{m}$ such that $\left(\gamma_{j}^{m}\right)^{6}=-\tilde{f}^{m}\left(\tau_{j}\right)+e_{m}\left(\tau_{j}\right)+2 \epsilon_{m}$. Because of (14) the supports of $p_{j}^{m}, j=1, \ldots, m$, are disjoint. Let $p^{m}=\sum_{j} p_{j}^{m}$, where the summation is for all $j$ such that either $d_{m}\left(\tau_{j}\right)-\tilde{f}^{m}\left(\tau_{j}\right)<\epsilon_{m}$ or $-e_{m}\left(\tau_{j}\right)+\tilde{f}^{m}\left(\tau_{j}\right)<\epsilon_{m}$. Note that $p^{m}(t)$ is a $C^{2}$ function and $\left(p^{m}\right)^{\prime \prime} \rightarrow 0 L^{2}$-strongly as $m \rightarrow \infty$. Define $\bar{f}^{m}=\tilde{f}^{m}+p^{m}$. By construction, for every $m$ sufficiently large, $\bar{f}^{m}$ is feasible for both problems (2) and (10). Moreover, $\lim _{m \rightarrow \infty}\left\|\left(\bar{f}^{m}\right)^{\prime \prime}-\left(f^{0}\right)^{\prime \prime}\right\|_{L^{2}}=0$.

The remaining part of the proof is standard. Since

$$
\left\|\left(f^{m}\right)^{\prime \prime}\right\|_{L^{2}} \leq\left\|\left(\bar{f}^{m}\right)^{\prime \prime}\right\|_{L^{2}} \rightarrow\left\|\left(f^{0}\right)^{\prime \prime}\right\|_{L^{2}}
$$


as $m \rightarrow \infty$, it follows that the sequence $\left\{\left(f^{m}\right)^{\prime \prime}\right\}$ is bounded in $L^{2}[0,1]$. Hence, one can extract a subsequence $\left\{\left(f^{m_{k}}\right)^{\prime \prime}\right\}$ which converges weakly to some $\hat{u} \in L^{2}[0,1]$. Define

$$
\hat{f}(t)=y_{1}+t\left(y_{n}-y_{1}-\int_{0}^{1}(t-\sigma) \hat{u}(\sigma) d \sigma\right)+\int_{0}^{t}(t-\sigma) \hat{u}(\tau) d \tau .
$$

Since $\left(f^{m_{k}}\right)^{\prime \prime} \rightarrow \hat{u} L^{2}$-weakly, $f^{m_{k}} \rightarrow \hat{f}$ in $C[0,1]$ as $m_{k} \rightarrow \infty$. Hence, $\hat{f}$ is feasible for problem (2) and because of the weak lower semicontinuity of the $L^{2}$-norm we have

$$
\left\|\left(f^{0}\right)^{\prime \prime}\right\|_{L^{2}} \leq\left\|\hat{f}^{\prime \prime}\right\|_{L^{2}} \leq \lim \inf _{m_{k} \rightarrow \infty}\left\|\left(f^{m_{k}}\right)^{\prime \prime}\right\|_{L^{2}} \leq \lim _{m_{k} \rightarrow \infty}\left\|\left(\bar{f}^{m_{k}}\right)^{\prime \prime}\right\|_{L^{2}}=\left\|\left(f^{0}\right)^{\prime \prime}\right\|_{L^{2}}
$$

Since the minimum of problem (2) is unique we obtain that $\left(f^{m_{k}}\right)^{\prime \prime} \rightarrow\left(f^{0}\right)^{\prime \prime} L^{2}$-weakly and $\left\|\left(f^{m_{k}}\right)^{\prime \prime}\right\|_{L^{2}} \rightarrow\left\|\left(f^{0}\right)^{\prime \prime}\right\|_{L^{2}}$ as $m_{k} \rightarrow \infty$. Hence, $\left(f^{m_{k}}\right)^{\prime \prime} \rightarrow\left(f^{0}\right)^{\prime \prime} L^{2}$-strongly. Using the representation (18) we complete the proof.

The transformation of the numerical approach outlined before Theorem 4.1 into a computational procedure requires further analysis supported by numerical examples; this is a subject of continuing research.

Remark 4.1. From Theorem 4.1 we immediately obtain the following result. Consider problem (2), where $e$ and $d$ are continuous, piecewise linear in every interval $\left(t_{i}, t_{i+1}\right)$, $i=1, \ldots, n$, and satisfy the interiority condition. Let the knots of $e$ and $d$ be $0=\tau_{1}<$ $\tau_{2}<\cdots<\tau_{m}=1,\left\{t_{1}, \ldots, t_{n}\right\} \subset\left\{\tau_{1}, \ldots, \tau_{m}\right\}$. Then, the unique solution $f$ of problem (2) is a $C^{1}$ piecewise cubic polynomial with knots $\tau_{j}, j=1, \ldots, m$, and no more than two additional knots in every interval $\left(\tau_{j}, \tau_{j+1}\right), j=1,2, \ldots, m-1$. In every interval $\left(\tau_{j}, \tau_{j+1}\right)$, the following cases are possible: the constraints are not active; there is one additional knot that is either a single touching point or an end of a subarc whose other end is either $\tau_{j}$ or $\tau_{j+1}$; there are two additional knots that either form a touching pair or are the ends of one subarc, or are the ends of two subarcs whose other ends are $\tau_{j}$ and $\tau_{j+1}$.

Remark 4.2. Suppose that in problem (2) the constraints $e$ and $d$ are continuous and satisfy inf $_{t \in[0.1]}|d(t)-e(t)|>0, e\left(t_{i}\right) \leq y_{i} \leq d\left(t_{i}\right), i=1, \ldots, n$, but the interpolation points are not necessarily strictly between $e$ and $d$; that is, either $e\left(t_{i}\right)=y_{i}$ or $d\left(t_{i}\right)=y_{i}$ for some $t_{i}$. For this case Lemma 1.1 does not apply. Assume that the feasible set is nonempty, i.e. there exists a function $\psi \in W^{2.2}[0,1]$ with $\psi\left(t_{i}\right)=y_{i}, i=1,2, \ldots, n$, such that $e(t) \leq \psi(t) \leq d(t)$, for all $t \in[0,1]$. Then problem (2) has a solution $f^{0}$ and the solution is unique, see [7]. Let $\left\{\epsilon_{m}\right\}$ be a sequence of positive numbers convergent to zero. Consider the following problem as an approximation of problem (2) for $m$ sufficiently large:

$$
\begin{aligned}
& \left\|f^{\prime \prime}\right\|_{L^{2}} \rightarrow \min , \\
& f\left(t_{i}\right)=y_{i}-\epsilon_{m}, \quad \text { if } y_{i}=d\left(t_{i}\right), \\
& f\left(t_{i}\right)=y_{i}+\epsilon_{m}, \quad \text { if } y_{i}=e\left(t_{i}\right), \\
& f\left(t_{i}\right)=y_{i}, \quad \text { if } e\left(t_{i}\right)<y_{i}<d\left(t_{i}\right), \\
& e(t) \leq f(t) \leq d(t) \text { for all } t \in[0,1] .
\end{aligned}
$$


Let $f^{m}$ be the unique solution of problem (19). Using the argument in the proof of Theorem 4.1 it can be shown that $f^{m} \rightarrow f^{0}$ in the norm of $W^{2,2}[0,1]$ as $m \rightarrow \infty$. From this result we obtain that if $e$ and $d$ are piecewise linear (cubic) across the grid $\left\{t_{i}\right\}$, then $f^{0}$ is a $C^{1}$ cubic spline with no more than two (four) additional knots in every interval $\left(t_{i}, t_{i+1}\right)$.

\section{Numerical examples}

We present numerical results for three examples. The first example illustrates the algorithm for the case when the constraints are continuous and linear in each interval $\left[t_{i}, t_{i+1}\right]$. The second example has discontinuous constraints that are linear in all intervals $\left(t_{i}, t_{i+1}\right)$ but one, where the lower constraint is cubic. The third example illustrates Remark 4.2. The BFGS code with approximation of the gradient from Optimization Toolbox and Spline Toolbox, both from Matlab 4.0 have been used in computations. As initial values of the derivatives $z_{i}$ at the knots $t_{i}$ we choose the derivatives of the function with minimal second norm of the second derivative that interpolates $\left(t_{i}, y_{i}\right)$ (the natural cubic spline). For all the examples the computational time was from 3 to 7 minutes on Hewlett Packard (HP) Workstation, 9000 Series, Model $715 / 50$ with a $50 \mathrm{MHz}$ PA-RISC 7100 Processor. The computations were terminated when the norm of a finite-difference approximation of the gradient of the objective function becomes less than $10^{-6}$.

Example 5.1. This example has 16 interpolating points $t_{i}$, shown by "o" in figures 1 and 2. The constraints $e$ and $d$ are indicated by the dashed lines. The constraints are continuous functions and linear in each interval $\left(t_{i}, t_{i+1}\right)$. The unconstrained cubic spline is shown by the solid line in figure 1 . It significantly violates the constraints. The constrained cubic spline is obtained by the method described in Section 3. It is shown by the solid line in figure 2 and has nine additional knots indicated by "*": five single touching points, one subarc in the 12th interval and one touching pair in the 14th interval. The $L^{2}$ norm of the second derivative of the unconstrained spline is 3.711 , while the $L^{2}$ norm of the second derivative of the constrained spline is 4.931 .

Example 5.2. The second example (see the unconstrained cubic spline in figure 3) has constraints that are discontinuous at several $t_{i}$ and the lower constraint is cubic in the fourth interval. There are also additional interpolation conditions on the end slopes: $f^{\prime}(0)=-2$, $f^{\prime}(16)=-2$. The specified end slope conditions force the unconstrained cubic spline significantly violate the constraints. From the development in Section 3 it is clear that the case of additional (Hermite-type) conditions for the slopes at the ends of the interval $[0,1]$ can be easily handled by the algorithm. The case when $e$ and $d$ are cubic in each interval $\left(t_{i}, t_{i+1}\right)$ can be treated similarly to the case when $e$ and $d$ are linear in each interval $\left(t_{i}, t_{i+1}\right)$. The solution has four subarcs and three touching points and is given in figure 4.

Example 5.3. This example illustrates the discussion in Remark 4.2 for the case where some interpolation points are on the boundary of the feasible region. Here $n=2, t_{1}=0$, $t_{2}=25, f(0)=0, f(25)=-1$ and there are additional constraints on the end slopes $f^{\prime}(0)=-2, f^{\prime}(25)=2$. The constraints $e$ and $d$ are indicated in figure 5 by the dashed 


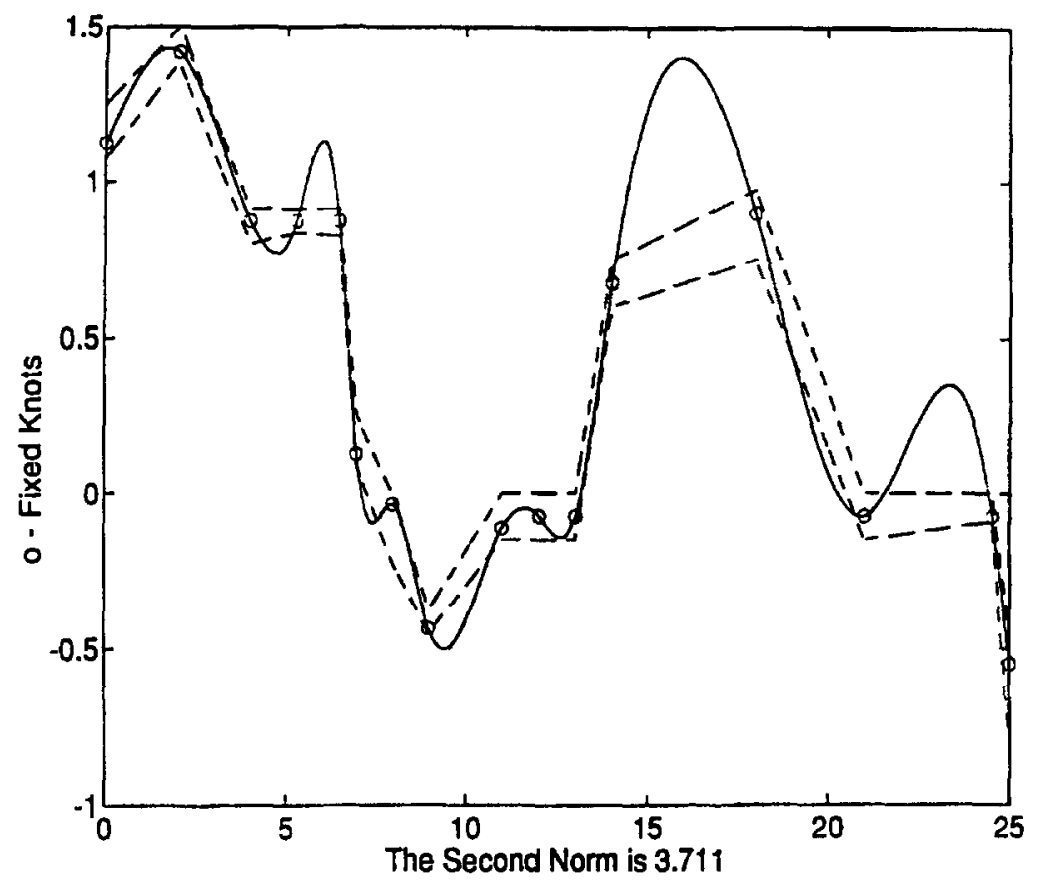

Figure 1. The unconstrained spline in Example 5.1.

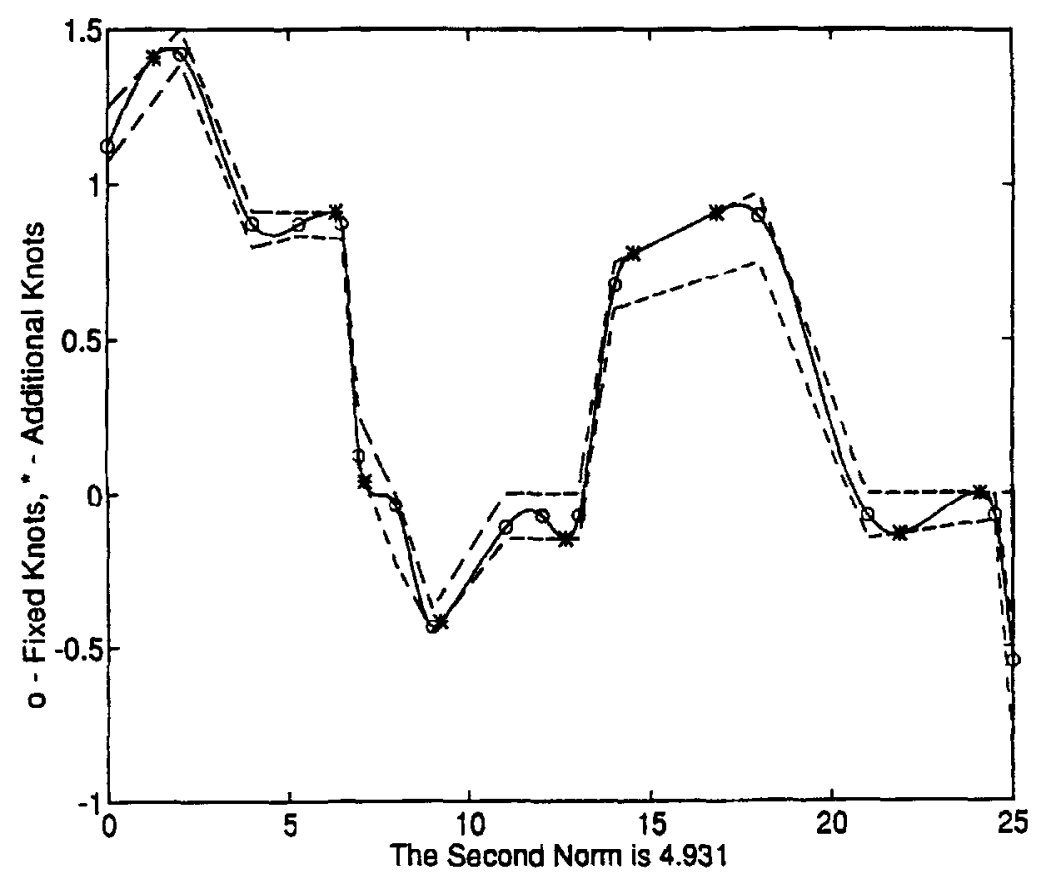

Figure 2. The constrained spline in Example 5.1. 


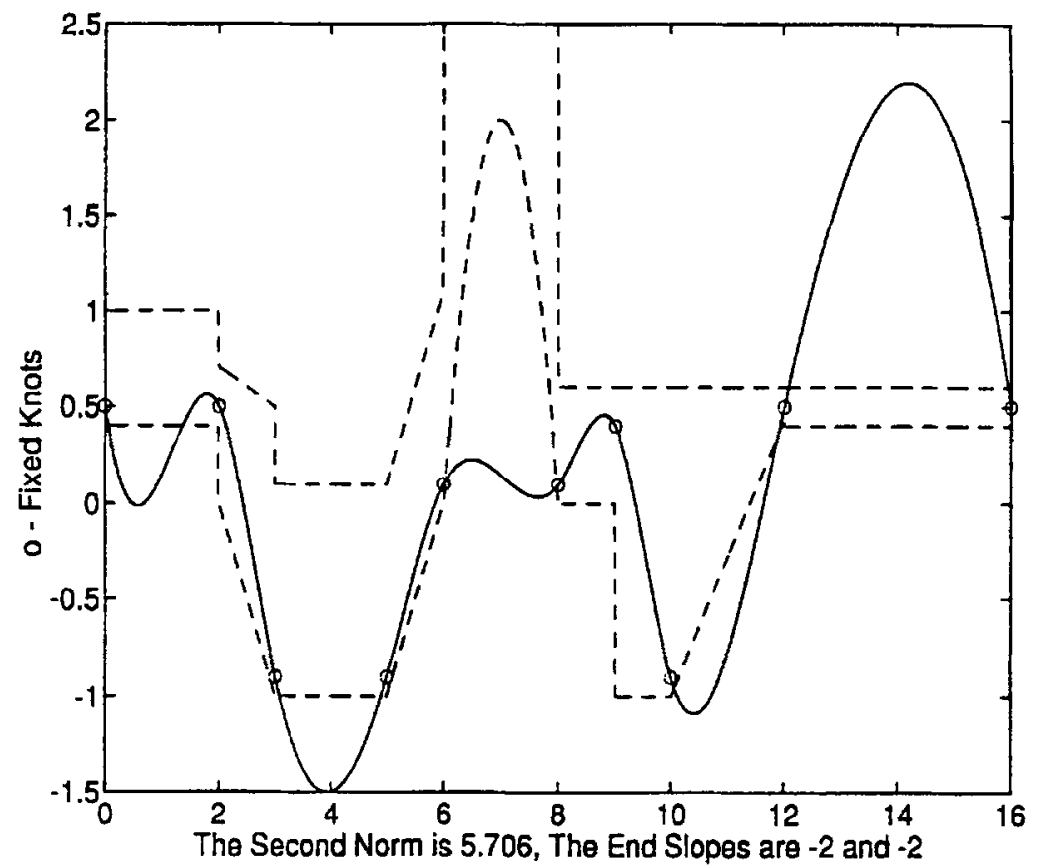

Figure 3. The unconstrained spline in Example 5.2.

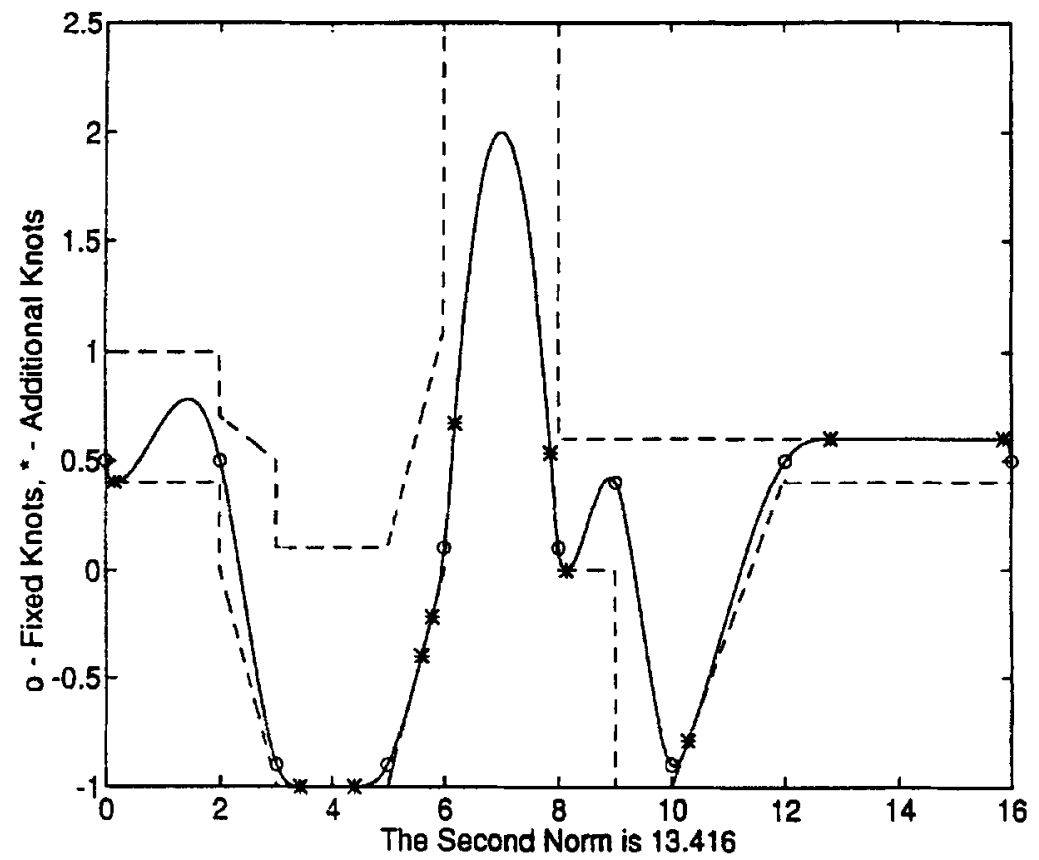

Figure 4. The constrained spline in Example 5.2. 


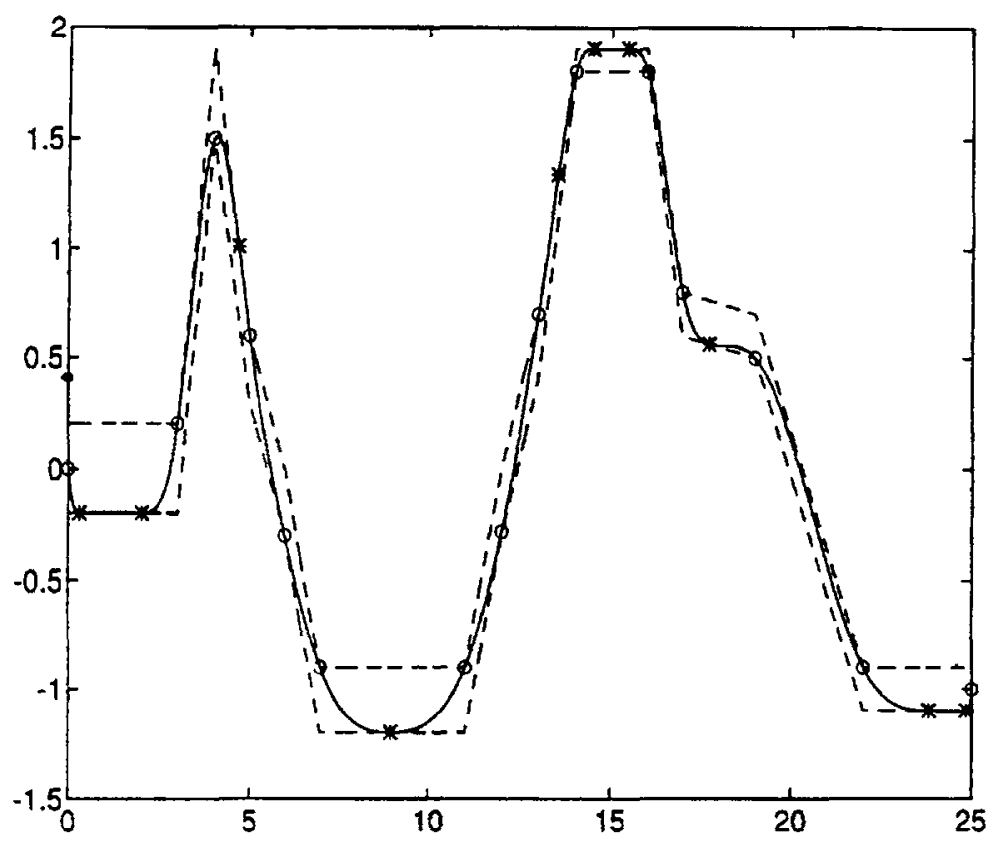

Figure 5. The constrained spline in Example 5.3.

lines. They are continuous and piecewise linear in $\left[t_{1}, t_{2}\right]$. The constrained spline is shown in Figure 5 by the solid line. The knots $\tau_{j}, j=1, \ldots, 15$, are indicated by " 0 " and the ten additional knots are indicated by “*”.

\section{References}

1. L.-E. Andersson and T. Elfving, "An algorithm for constrained interpolation," SIAM J. Sci. Stat. Comput., vol. 8, pp. 1012-025, 1987

2. L.-E. Andersson and T. Elfving, "Best constrained approximation in Ililbert space and interpolation by cubic splines subject to obstacles," SiAM J. Sci. Stat. Comput., vol. 16, pp. 1209-1232, 1995.

3. C. de Boor, "On "best" interpolation," J. Approx. Theory, vol. 16, pp. 28-42, 1976.

4. J.M. Borwein and A.S. Lewis, "Partially finite convex programming," Mathematical Programming. vol. 57. 1992, "Part I: Quasi relative interiors and duality theory," pp. 15-48; "Part II: Explicit lattice models," pp. 49-83.

5. C.K. Chui, F. Deutsch, and J.D. Ward, "Constrained best approximation in Hilbert space," Part I: Constr. Approx., vol. 6, pp. 35-64, 1990; Part II: J. Approx. Theory, vol. 71, pp. 213-238, 1992.

6. A.L. Dontchev, "Duality methods for constrained best interpolation," Math. Balkanica, vol. 1, pp. 96-105, 1987.

7. A.L. Dontchev, "Best interpolation in a strip,” J. Approx. Theory, vol. 73, pp. 334-342, 1993.

8. A.L. Dontchev and B.D. Kalchev, "Duality and well-posedness in convex interpolation," Numer. Func. Anal. Optim., vol. 10, pp. 637-687, 1989.

9. A. I. Dontchev and I. Kolmanovsky, "State constraints in the linear regulator problem: A case study," J. Opt. Th. Appl., vol. 87, pp. 323-347, 1995.

10. B. Fischer, G. Opfer, and M.L. Puri, "A local algorithm for constructing non-negative cubic splines," J. Approx. Theory, vol. 64, pp. 1-16, 1991. 
11. J.C. Holladey, “A smoothest curvature approximation," Math. Tables $\Lambda$ ids Comput., vol. 11, pp. 233-243, 1957.

12. U. Hornung, "Interpolation by smooth functions under restrictions on the derivatives," J. Approx. Theory, vol. 28, pp. 227-237, 1980.

13. L.D. Irvine, S.P. Marin, and P.W. Smith, "Constrained interpolation and smoothing," Constructive Approx., vol. 2, pp. 129-151, 1986.

14. C.A. Micchelli, P.W. Smith, J. Swetits, and J.D. Ward, "Constrained $L_{p}$ approximation," Constr. Approx., vol. 1, pp. 93-102, 1985.

15. C.A. Micchelli and F.I. Utreras, "Smoothing and interpolation in a convex subset of a Hilbert space," SIAM J. Sci. Stat. Comput., vol. 9, pp. 728-746, 1988.

16. G. Opfer and H.J. Oberle, "The derivation of cubic splines with obstacles by methods of optimization and optimal control," Numer. Math., vol. 52, pp. 17-31, 1988. 